\title{
Multistage Spectral Relaxation Method for Solving the Hyperchaotic Complex Systems
}

\author{
Hassan Saberi Nik ${ }^{1}$ and Paulo Rebelo ${ }^{2}$ \\ ${ }^{1}$ Department of Mathematics, Islamic Azad University, Mashhad Branch, Mashhad, Iran \\ ${ }^{2}$ Departamento de Matemática, Universidade da Beira Interior, 6201-001 Covilhã, Portugal \\ Correspondence should be addressed to Hassan Saberi Nik; saberi_hssn@yahoo.com
}

Received 4 August 2014; Accepted 12 September 2014; Published 16 October 2014

Academic Editor: Fazlollah Soleymani

Copyright (C) 2014 H. Saberi Nik and P. Rebelo. This is an open access article distributed under the Creative Commons Attribution License, which permits unrestricted use, distribution, and reproduction in any medium, provided the original work is properly cited.

We present a pseudospectral method application for solving the hyperchaotic complex systems. The proposed method, called the multistage spectral relaxation method (MSRM) is based on a technique of extending Gauss-Seidel type relaxation ideas to systems of nonlinear differential equations and using the Chebyshev pseudospectral methods to solve the resulting system on a sequence of multiple intervals. In this new application, the MSRM is used to solve famous hyperchaotic complex systems such as hyperchaotic complex Lorenz system and the complex permanent magnet synchronous motor. We compare this approach to the Runge-Kutta based ode45 solver to show that the MSRM gives accurate results.

\section{Introduction}

Chaos theory studies the behaviour of dynamical systems that are highly sensitive to initial conditions and have complex and highly unpredictable profiles $[1,2]$. Chaotic systems can be observed in a wide variety of applications. In 1982, the complex Lorenz equations were proposed by Fowler et al. [3], which extended nonlinear systems into complex space. After that, some research works in this field have been achieved [4-9]. With in-depth study of complex nonlinear systems, a variety of physical phenomena could be described by the chaotic or hyperchaotic complex systems, for instance, the detuned laser systems and the amplitudes of electromagnetic fields.

The nature of complex chaotic systems precludes the possibility of obtaining closed form analytical solutions of the underlying governing equations. Thus, approximateanalytical methods, which are implemented on a sequence of multiple intervals to increase their radius of convergence, are often used to solve IVPs modelling chaotic systems. Examples of multistage methods that have been developed recently to solve IVPs for chaotic and nonchaotic systems include the multistage homotopy analysis method [10], piecewise homotopy perturbation methods [11, 12], multistage variational iteration method [13], and multistage differential transformation method [14]. Other multistage methods which use numerical integration techniques have also been proposed such as the piecewise spectral homotopy analysis method [15-17] which uses a spectral collocation method to perform the integration process. Accurate solutions of highly chaotic and hyperchaotic systems require resolution over many small intervals. Thus, seeking analytical solutions over the numerous intervals may be impractical or computationally expensive if the solution is sought over very long intervals.

In this paper, we propose a piecewise or multistage spectral relaxation method (MSRM) for solving the hyperchaotic complex systems as an accurate and robust alternative to recent multistage methods. The proposed MSRM was developed using the Gauss-Seidel idea of decoupling systems of equations and using Chebyshev pseudospectral methods to solve the resulting decoupled system on a sequence of multiple intervals. The spectral relaxation method (SRM) was recently proposed in $[18,19]$.

The rest of the paper is organized as follows. In Section 2, we give a brief description of the proposed MSRM algorithm. 
In Section 3, we present the numerical implementation of the MSRM on two examples of hyperchaotic complex systems. Finally, the conclusion is given in Section 4.

\section{Multistage Spectral Relaxation Method}

In this section, we give a brief description of the numerical method of solution used to solve the nonlinear hyperchaotic complex. We employ the multistage spectral relaxation method (MSRM) proposed in [19]. The MSRM algorithm is based on a Gauss-Seidel type of relaxation that decouples and linearises the system and the use of spectral collocation method to solve the linearised equations in a sequential manner. For compactness, we express the system of $m$ nonlinear first order differential equations in the form

$$
\begin{aligned}
\dot{x}_{r}(t)= & \sum_{k=1}^{m} \alpha_{r, k} x_{k}(t) \\
& +f_{r}\left[x_{1}(t), x_{2}(t), \ldots, x_{r-1}(t),\right. \\
& \left.x_{r+1}(t), \ldots, x_{m}(t)\right],
\end{aligned}
$$

subject to the initial conditions

$$
x_{r}(0)=x_{r}^{*}, \quad r=1,2, \ldots, m,
$$

where $x_{r}$ are the unknown variables and $x_{r}^{*}$ are the corresponding initial conditions, $\alpha_{r, k}$ are known constant input parameters and $f_{r}$ is the nonlinear component of the $r$ th equation and the dot denotes differentiation with respect to time $t$.

The scheme computes the solution of (1) in a sequence of equal subintervals that makes the entire interval. We define the interval of integration as $\Omega=[0, T]$ and divide it into a sequence of nonoverlapping subintervals $\Omega_{i}=$ $\left[t_{i-1}, t_{i}\right](i=1,2,3, \ldots, f)$, where $t_{0}=0$ and $t_{f}=T$. We denote the solution of (1) in the first subinterval $\left[t_{0}, t_{1}\right]$ as $x_{r}^{1}(t)$ and the solutions in the subsequent subintervals $\left[t_{i-1}, t_{i}\right](i=2,3, \ldots, f)$ as $x_{r}^{i}(t)$. For obtaining the solution in the first interval $\left[t_{0}, t_{1}\right],(2)$ is used as the initial condition. By using the continuity condition between neighbouring subintervals the obtained solution in the interval $\left[t_{0}, t_{1}\right]$ is used to obtain the initial condition for the next subinterval $\left[t_{1}, t_{2}\right]$. This is applied over the $f$ successive subintervals; that is, the obtained solution for each subinterval $\left[t_{i-1}, t_{i}\right]$ is used to obtain the initial condition for the next subinterval $\left[t_{i}, t_{i+1}\right](i=1,2, \ldots, f-1)$. Thus, in each interval $\left[t_{i-1}, t_{i}\right]$ we must solve

$$
\begin{aligned}
\dot{x}_{r}^{i}= & \alpha_{r, r} x_{r}^{i}+\left(1-\delta_{r s}\right) \sum_{k=1}^{m} \alpha_{r, k} x_{k}^{i} \\
& +f_{r}\left[x_{1}^{i}, \ldots, x_{r-1}^{i}, x_{r+1}^{i}, \ldots, x_{n}^{i}\right],
\end{aligned}
$$

subject to

$$
x_{r}^{i}\left(t_{i-1}\right)=x_{r}^{i-1}\left(t_{i-1}\right)
$$

where $\delta_{r s}$ is the Kronecker delta. As mentioned earlier, the main idea behind the MSRM scheme is decoupling the system of nonlinear IVPs using the Gauss-Seidel idea of decoupling systems of algebraic equations. The proposed MSRM iteration scheme for the solution in the interval $\Omega_{i}=$ $\left[t_{i-1}, t_{i}\right]$ is given as

$$
\begin{aligned}
& \dot{x}_{1, s+1}^{i}-\alpha_{1,1} x_{1, s+1}^{i}= \alpha_{1,2} x_{2, s}^{i}+\alpha_{1,3} x_{3, s}^{i} \\
&+\cdots+\alpha_{1, n} x_{n, s}^{i}+f_{1}\left[x_{1, s}^{i}, \ldots, x_{n, s}^{i}\right], \\
& \dot{x}_{2, s+1}^{i}-\alpha_{2,2} x_{2, s+1}^{i}= \alpha_{2,1} x_{1, s+1}^{i}+\alpha_{2,3} x_{3, s}^{i} \\
&+\cdots+\alpha_{2, n} x_{n, s}^{i} \\
&+f_{2}\left[x_{1, s+1}^{i}, x_{2, s}^{i}, \ldots, x_{n, s}^{i}\right] \\
& \vdots \\
&= \alpha_{m, 1} x_{1, s+1}^{i}+\cdots+\alpha_{m, m-1} x_{m-1, s+1}^{i} \\
&+f_{m}\left[x_{1, s+1}^{i}, \ldots, x_{m-1, s+1}^{i}, x_{m, s}^{i}\right] \\
&\left.\dot{x}_{m, s+1}^{i}-\alpha_{m, m} x_{m, s+1}^{i}\right]
\end{aligned}
$$

subject to the initial conditions

$$
x_{r, s+1}^{i}\left(t_{i-1}\right)=x_{r}^{i-1}\left(t_{i-1}\right), \quad r=1,2, \ldots, m,
$$

where $x_{r, s}$ is the estimate of the solution after $s$ iterations. A suitable initial guess to start the iteration scheme (5) is one that satisfies the initial condition (6). A convenient choice of initial guess that was found to work in the numerical experiments considered in this work is

$$
x_{r, 0}^{i}(t)= \begin{cases}x_{r}^{*} & \text { if } i=1, \\ x_{r}^{i-1}\left(t_{i-1}\right) & \text { if } 2 \leq i \leq f .\end{cases}
$$

The Chebyshev spectral method is used to solve (5) on each interval $\left[t_{i-1}, t_{i}\right]$. First, the region $\left[t_{i-1}, t_{i}\right]$ is transformed to the interval $[-1,1]$ on which the spectral method is defined by using the linear transformation,

$$
t=\frac{\left(t_{i}-t_{i-1}\right) \tau}{2}+\frac{\left(t_{i}+t_{i-1}\right)}{2},
$$

in each interval $\left[t_{i-1}, t_{i}\right]$ for $i=1, \ldots, f$. We then discretize the interval $\left[t_{i-1}, t_{i}\right]$ using the Chebyshev-Gauss-Lobatto collocation points [20]:

$$
\tau_{j}^{i}=\cos \left(\frac{\pi j}{N}\right), \quad j=1,2, \ldots, N,
$$

which are the extrema of the Nth order Chebyshev polynomial:

$$
T_{N}(\tau)=\cos \left(N \cos ^{-1} \tau\right)
$$

The Chebyshev spectral collocation method is based on the idea of introducing a differentiation matrix $D$ which is used to approximate the derivatives of the unknown variables $x_{r, s+1}^{i}(t)$ at the collocation points as the matrix vector product

$$
\left.\frac{d x_{r, s+1}^{i}}{d t}\right|_{t=t_{j}}=\sum_{k=0}^{N} \mathbf{D}_{j k} x_{r, s+1}^{i}=\mathbf{D X}_{r, s+1}^{i}, \quad j=1,2, \ldots, N,
$$


TABLE 1: Numerical comparison between MSRM and ode45 for the hyperchaotic complex Lorenz system.

\begin{tabular}{lcccccc}
\hline$t$ & & $x_{1}(t)$ & & $x_{2}(t)$ & & $x_{3}(t)$ \\
MSRM & & ode45 & MSRM & ode45 & MSRM & -3.24491 \\
\hline 2 & -2.91138 & -2.91138 & 21.73155 & 21.73155 & -3.24491 & -6.30884 \\
4 & -3.63001 & -3.63001 & 6.52144 & 6.52144 & -6.30884 & -2.37099 \\
6 & 2.80571 & 2.80571 & -2.77638 & -2.77638 & -2.37099 & -0.14880 \\
8 & 0.01134 & 0.01134 & 2.09585 & 2.09585 & -0.14880 & -0.06690 \\
10 & -0.80219 & -0.80219 & 16.48559 & 16.48560 & -0.06690 & \\
\hline
\end{tabular}

where $\mathbf{D}=2 \mathrm{D} /\left(t_{i}-t_{i-1}\right)$ and $\mathbf{X}_{r, s+1}^{i}=\left[x_{r, s+1}^{i}\left(\tau_{0}^{i}\right), x_{r, s+1}^{i}\left(\tau_{1}^{i}\right)\right.$, $\left.\ldots, x_{r, s+1}^{i}\left(\tau_{N}^{i}\right)\right]$ are the vector functions at the collocation points $\tau_{j}^{i}$.

Applying the Chebyshev spectral collocation method in (5) gives

$$
\begin{aligned}
\mathbf{A}_{r} \mathbf{X}_{r, s+1}^{i}=\mathbf{B}_{r}^{i}, \quad \mathbf{X}_{r, s+1}^{i}\left(\tau_{N}^{i-1}\right) & =\mathbf{X}_{r}^{i-1}\left(\tau_{N}^{i-1}\right), \\
r & =1,2, \ldots, m,
\end{aligned}
$$

with

$$
\begin{aligned}
\mathbf{A}_{r}= & \mathbf{D}-\alpha_{r, r} \mathbf{I} \\
\mathbf{B}_{1}^{i}= & \alpha_{1,2} \mathbf{X}_{2, s}^{i} \\
& +\cdots+\alpha_{1, n} \mathbf{X}_{n, s}^{i}+f_{1}\left[\mathbf{X}_{1, s}^{i}, \ldots, \mathbf{X}_{m, s}^{i}\right] \\
\mathbf{B}_{2}^{i}= & \alpha_{2,1} \mathbf{X}_{1, s+1}^{i}+\alpha_{2,3} \mathbf{X}_{3, s}^{i} \\
& +\cdots+\alpha_{2, m} \mathbf{X}_{m, s}^{i}+f_{2}\left[\mathbf{X}_{1, s+1}^{i}, \mathbf{X}_{2, s}^{i}, \ldots, \mathbf{X}_{m, s}^{i}\right] \\
\vdots & \quad \\
\mathbf{B}_{m}^{i}= & \alpha_{m, 1} \mathbf{X}_{1, s+1}^{i}+\alpha_{m, 2} \mathbf{X}_{2, s+1}^{i}+\cdots+\alpha_{m, m-1} \mathbf{X}_{m-1, s+1}^{i} \\
& +f_{m}\left[\mathbf{X}_{1, s+1}^{i}, \ldots, \mathbf{X}_{m-1, s+1}^{i}, \mathbf{X}_{m, s}^{i}\right]
\end{aligned}
$$

where $\mathbf{I}$ is an identity matrix of order $N+1$. Thus, starting from the initial approximation (7), the recurrence formula

$$
\mathbf{X}_{r, s+1}^{i}=\mathbf{A}_{r}^{-1} \mathbf{B}_{r}^{i}, \quad r=1,2, \ldots, m
$$

can be used to obtain the solution $x_{r}^{i}(t)$ in the interval $\left[t_{i-1}, t_{i}\right]$. The solution approximating $x_{r}(t)$ in the entire interval $\left[t_{0}, t_{F}\right]$ is given by

$$
x_{r}(t)= \begin{cases}x_{r}^{1}(t), & t \in\left[t_{0}, t_{1}\right] \\ x_{r}^{2}(t), & t \in\left[t_{1}, t_{2}\right] \\ \vdots & \\ x_{r}^{F}(t), & t \in\left[t_{f-1}, t_{f}\right] .\end{cases}
$$

\section{Numerical Examples}

In this section, we consider two examples which demonstrate the efficiency and accuracy of the proposed method. In particular, we use the MSRM algorithm as an appropriate tool for solving nonlinear IVPs; we apply the method to two complex nonlinear chaotic systems.
Example 1. The hyperchaotic complex Lorenz system can be described as

$$
\begin{aligned}
& \dot{z}_{1}=a_{1}\left(z_{2}-z_{1}\right)+j z_{4}, \\
& \dot{z}_{2}=a_{2} z_{1}-z_{2}-z_{1} z_{3}+j z_{4}, \\
& \dot{z}_{3}=\frac{1}{2}\left(z_{1} \bar{z}_{2}+\bar{z}_{1} z_{2}\right)-a_{3} z_{3}, \\
& \dot{z}_{4}=\frac{1}{2}\left(z_{1} \bar{z}_{2}+\bar{z}_{1} z_{2}\right)-a_{4} z_{4},
\end{aligned}
$$

where $z_{1}=x_{1}+j x_{2}, z_{2}=x_{3}+j x_{4}, z_{3}=x_{5}, z_{4}=x_{6}$, $j=\sqrt{-1}, \bar{z}_{1}$ and $\bar{z}_{2}$ are the conjugates of $z_{1}$ and $z_{2}$. When the parameters are chosen as $a_{1}=15, a_{2}=36, a_{3}=4.5$, and $a_{4}=12$, the system (16) is hyperchaotic [21].

Replacing the complex variables in system (16) with real and imaginary number variables, one can get an equivalent system as follows:

$$
\begin{aligned}
& \dot{x}_{1}=a_{1}\left(x_{3}-x_{1}\right), \\
& \dot{x}_{2}=a_{1}\left(x_{4}-x_{2}\right)+x_{6}, \\
& \dot{x}_{3}=a_{2} x_{1}-x_{3}-x_{1} x_{5}, \\
& \dot{x}_{4}=a_{2} x_{2}-x_{4}-x_{2} x_{5}+x_{6}, \\
& \dot{x}_{5}=x_{1} x_{3}+x_{2} x_{4}-a_{3} x_{5}, \\
& \dot{x}_{6}=x_{1} x_{3}+x_{2} x_{4}-a_{4} x_{6} .
\end{aligned}
$$

For (17), the parameters $\alpha_{r, k}$ and $f_{r}$ are defined as

$$
\begin{gathered}
\alpha_{1,1}=-a_{1}, \quad \alpha_{1,3}=a_{1}, \quad \alpha_{2,2}=-a_{1}, \\
\alpha_{2,4}=a_{1}, \quad \alpha_{2,6}=1, \\
\alpha_{3,1}=a_{2}, \quad \alpha_{3,3}=-1, \quad \alpha_{4,2}=a_{2}, \\
\alpha_{4,4}=-1, \quad \alpha_{4,6}=1, \\
\alpha_{5,5}=-a_{3}, \quad \alpha_{6,6}=-a_{4}, \quad f_{3}=-x_{1} x_{5}, \\
f_{4}=-x_{2} x_{5}, \quad f_{5}=f_{6}=x_{1} x_{3}+x_{2} x_{4},
\end{gathered}
$$

with all other $\alpha_{r, k}$ and $f_{r}=0$ for $r, k=1,2, \ldots, 6$.

Through numerical experimentation, it was determined that $N=6$ collocation points and 5 iterations of the MSRM scheme at each interval were sufficient to give accurate results in each $\left[t_{i-1}, t_{i}\right]$ interval. Tables 1 and 2 show a comparison of the solutions of the hyperchaotic complex Lorenz system 
TABLE 2: Numerical comparison between MSRM and ode45 for the hyperchaotic complex Lorenz system.

\begin{tabular}{|c|c|c|c|c|c|c|}
\hline \multirow{2}{*}{$t$} & \multicolumn{2}{|c|}{$x_{4}(t)$} & \multicolumn{2}{|c|}{$x_{5}(t)$} & \multicolumn{2}{|c|}{$x_{6}(t)$} \\
\hline & MSRM & ode 45 & MSRM & ode 45 & MSRM & ode 45 \\
\hline 2 & 23.96851 & 23.96851 & 44.32071 & 44.32071 & 26.54682 & 26.54682 \\
\hline 4 & 11.30830 & 11.30830 & 14.68007 & 14.68007 & 3.25221 & 3.25221 \\
\hline 6 & 4.65208 & 4.65208 & 39.34559 & 39.34559 & 12.99055 & 12.99055 \\
\hline 8 & -4.99685 & -4.99685 & 33.79560 & 33.79560 & 8.02232 & 8.02232 \\
\hline 10 & 1.98179 & 1.98179 & 50.59739 & 50.59740 & 24.48234 & 24.48234 \\
\hline
\end{tabular}

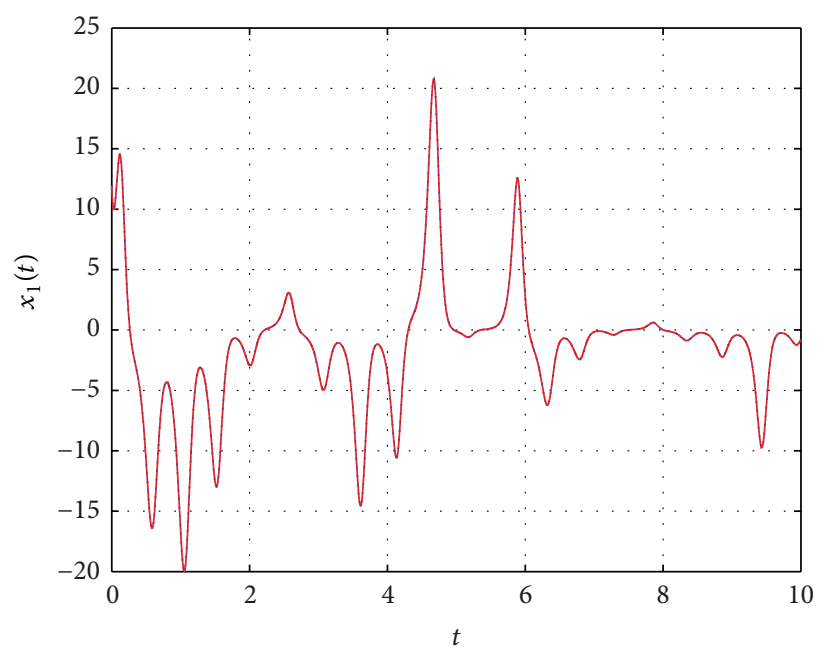

- MSRM

ode 45

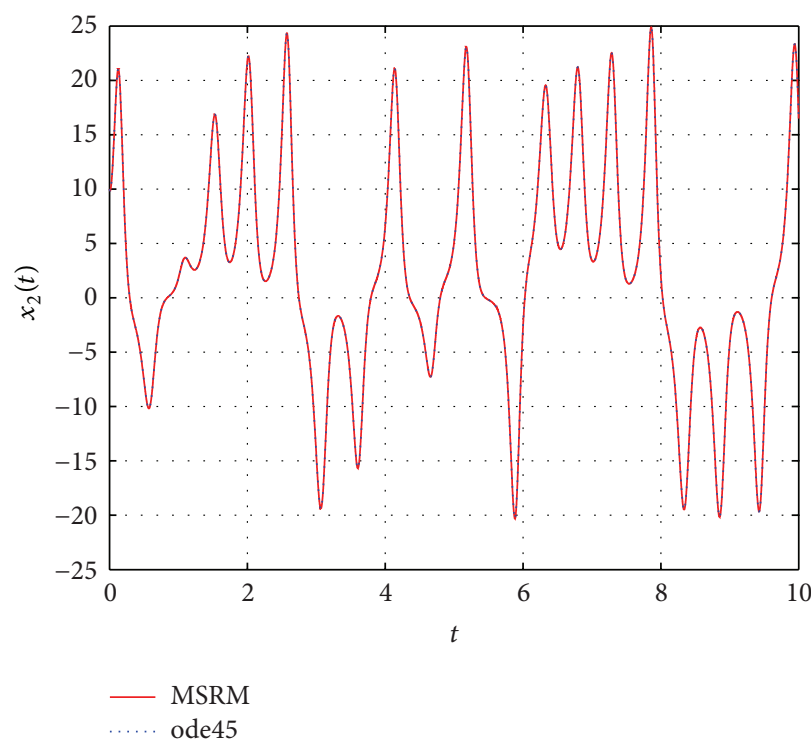

FIGURE 1: Comparison between the MSRM and ode45 results for the hyperchaotic complex Lorenz system. computed by the MSRM and ode45. In Figures 1, 2, and 3, the MSRM graphical results are also compared with ode45 and good agreement is observed. The MRSM phase portraits in Figures 4 and 5 were also found to be exactly the same as those computed using ode45. This shows that the proposed MSRM is a valid tool for solving the hyperchaotic complex Lorenz system.

Example 2. State equations of a permanent magnet synchronous motor system in a field-oriented rotor can be described as follows $[22,23]$ :

$$
\begin{aligned}
& \frac{d i_{d}}{d t}=\frac{-R_{1} i_{d}+\omega L_{q} i_{q}+u_{d}}{L_{d}}, \\
& \frac{d i_{q}}{d t}=\frac{R_{1} i_{q}+\omega L_{d} i_{q}+u_{q}-\omega \Psi_{r}}{L_{q}}, \\
& \frac{d \omega}{d t}=\frac{n_{q} \Psi_{r} i_{d}+n_{p}\left(L_{d}-L_{q}\right) i_{d} i_{q}-T_{L}-\beta \omega}{J},
\end{aligned}
$$

where $i_{d}, i_{q}$, and $\omega$ are the state variables which represent currents and motor angular frequency, respectively; $u_{d}$ and $u_{q}$ are the direct-axis stator and quadrature-axis stator voltage components, respectively; $J$ is the polar moment of inertia; $T_{L}$ is the external load torque; $\beta$ is the viscous damping coefficient; $R_{1}$ is the stator winding resistance; $L_{d}$ and $L_{q}$ are the direct-axis stator inductors and quadrature-axis stator inductors, respectively; $\Psi_{r}$ is the permanent magnet flux; and $n_{p}$ is the number of pole-pairs; the parameters $L_{d}, L_{q}, J, T_{L}$, $R_{1}, \Psi_{r}, \beta$ are all positive.

When the air gap is even, and the motor has no load or power outage, the dimensionless equations of a permanent magnet synchronous motor system can be depicted as

$$
\begin{aligned}
& \dot{z}_{1}=a\left(z_{2}-z_{1}\right), \\
& \dot{z}_{2}=b z_{1}-z_{2}-z_{1} z_{3}, \\
& \dot{z}_{3}=z_{1} z_{2}-z_{3},
\end{aligned}
$$

where $a, b$ are both positive parameters. If the current in the system (19) is plural and the variables $z_{1}, z_{2}$ in the system (20) are complex numbers, by changing cross coupled terms $z_{1}$ and $z_{2}$ to conjugate form, Wang and Zhang got a complex 

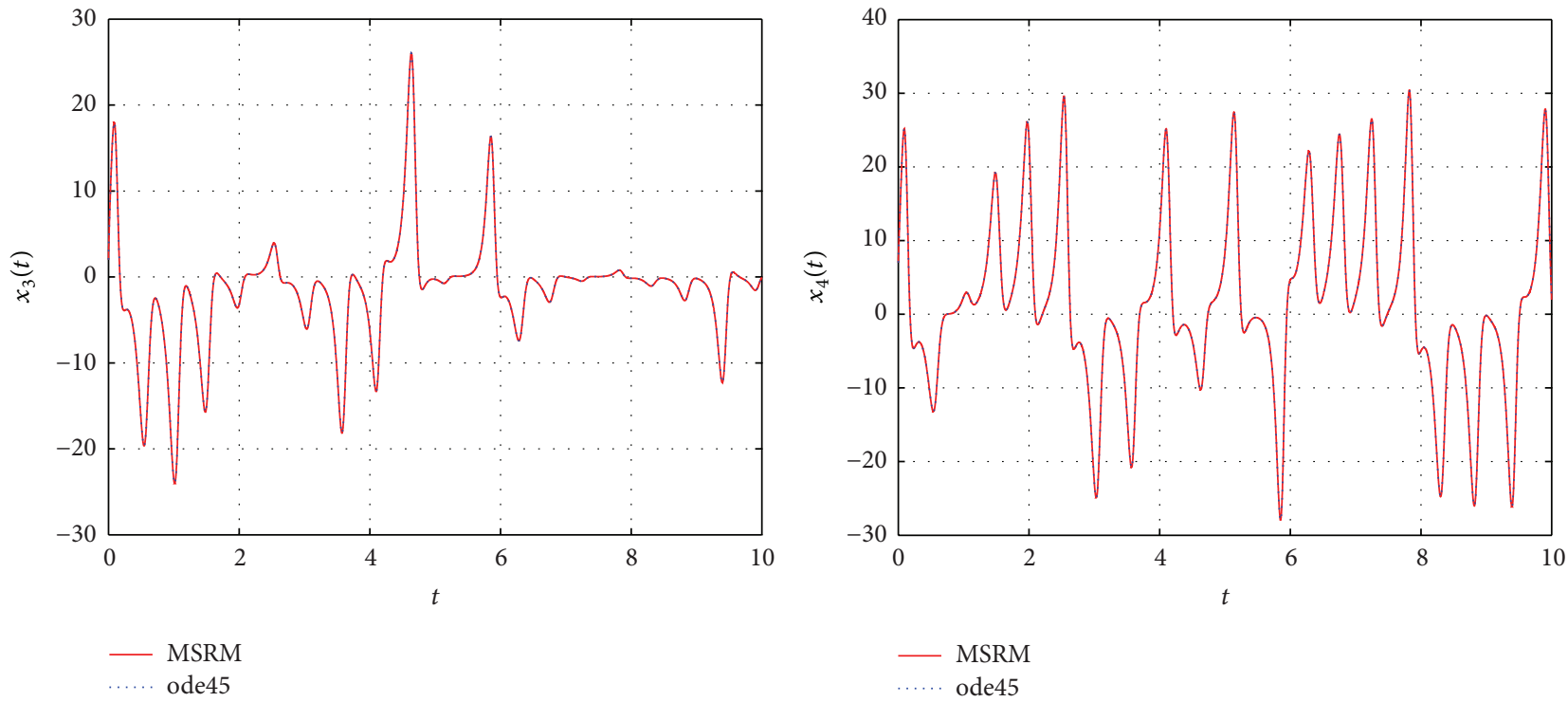

Figure 2: Comparison between the MSRM and ode45 results for the hyperchaotic complex Lorenz system.
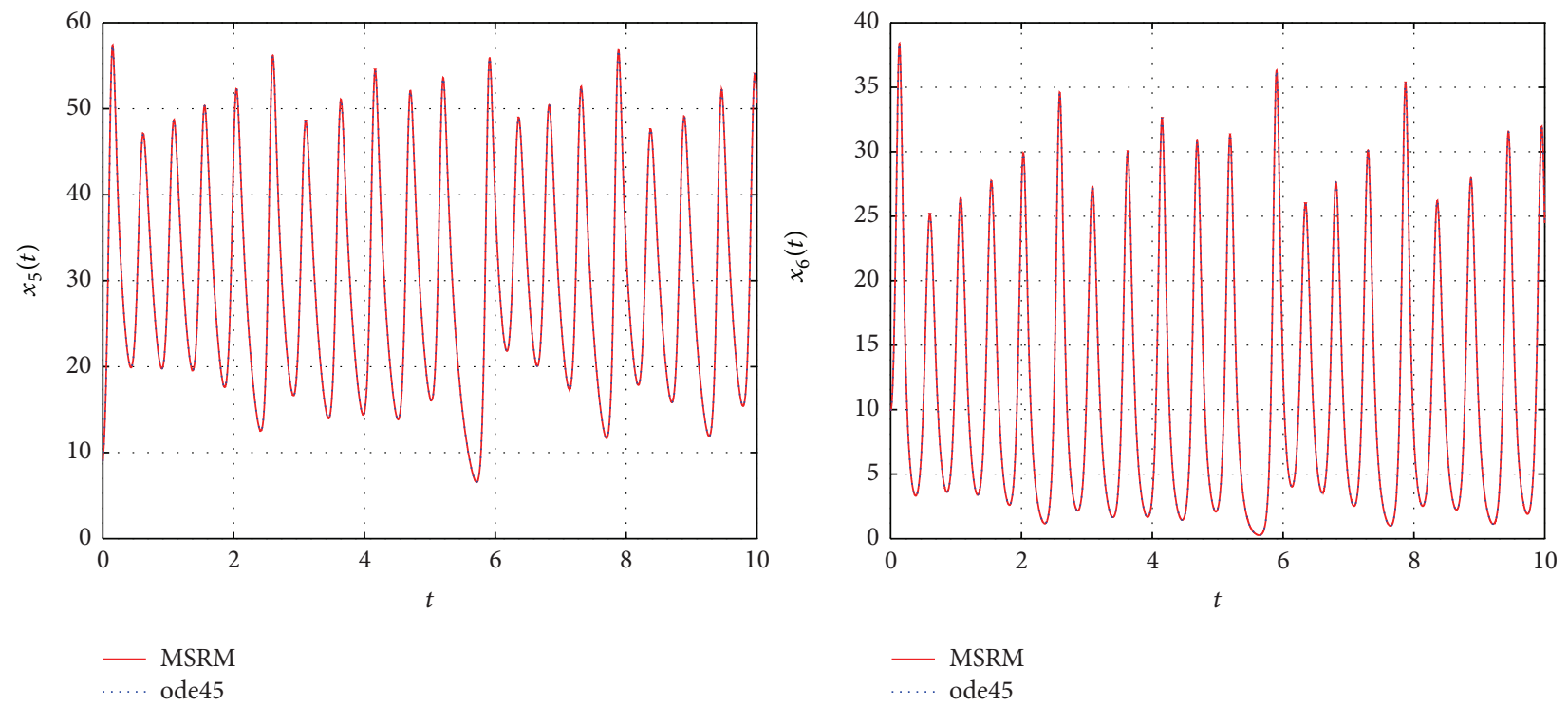

FIGURE 3: Comparison between the MSRM and ode45 results for the hyperchaotic complex Lorenz system.

permanent magnet synchronous motor system as follows [24]:

$$
\begin{aligned}
& \dot{z}_{1}=a\left(z_{2}-z_{1}\right), \\
& \dot{z}_{2}=b z_{1}-z_{2}-z_{1} z_{3}, \\
& \dot{z}_{3}=\frac{1}{2}\left(z_{1} \bar{z}_{2}+\bar{z}_{1} z_{2}\right)-z_{3},
\end{aligned}
$$

where $z_{1}=x_{1}+j x_{2}, z_{2}=x_{3}+j x_{4}, z_{3}=x_{5}, j=\sqrt{-1}$, $\bar{z}_{1}$ and $\bar{z}_{2}$ are the conjugates of $z_{1}$ and $z_{2}$. Replacing the complex variables in system (21) with real and imaginary number variables, Wang and Zhang got an equivalent system as follows (see [24]):

$$
\begin{aligned}
& \dot{x}_{1}=a\left(x_{3}-x_{1}\right), \\
& \dot{x}_{2}=a\left(x_{4}-x_{2}\right), \\
& \dot{x}_{3}=b x_{1}-x_{3}-x_{1} x_{5}, \\
& \dot{x}_{4}=b x_{2}-x_{4}-x_{2} x_{5}, \\
& \dot{x}_{5}=x_{1} x_{3}+x_{2} x_{4}-x_{5},
\end{aligned}
$$

where $a, b$ are positive parameters determining the chaotic behaviors and bifurcations of system (22). When the parameters satisfy $1 \leq a \leq 11,10 \leq b \leq 20$, there is one positive 

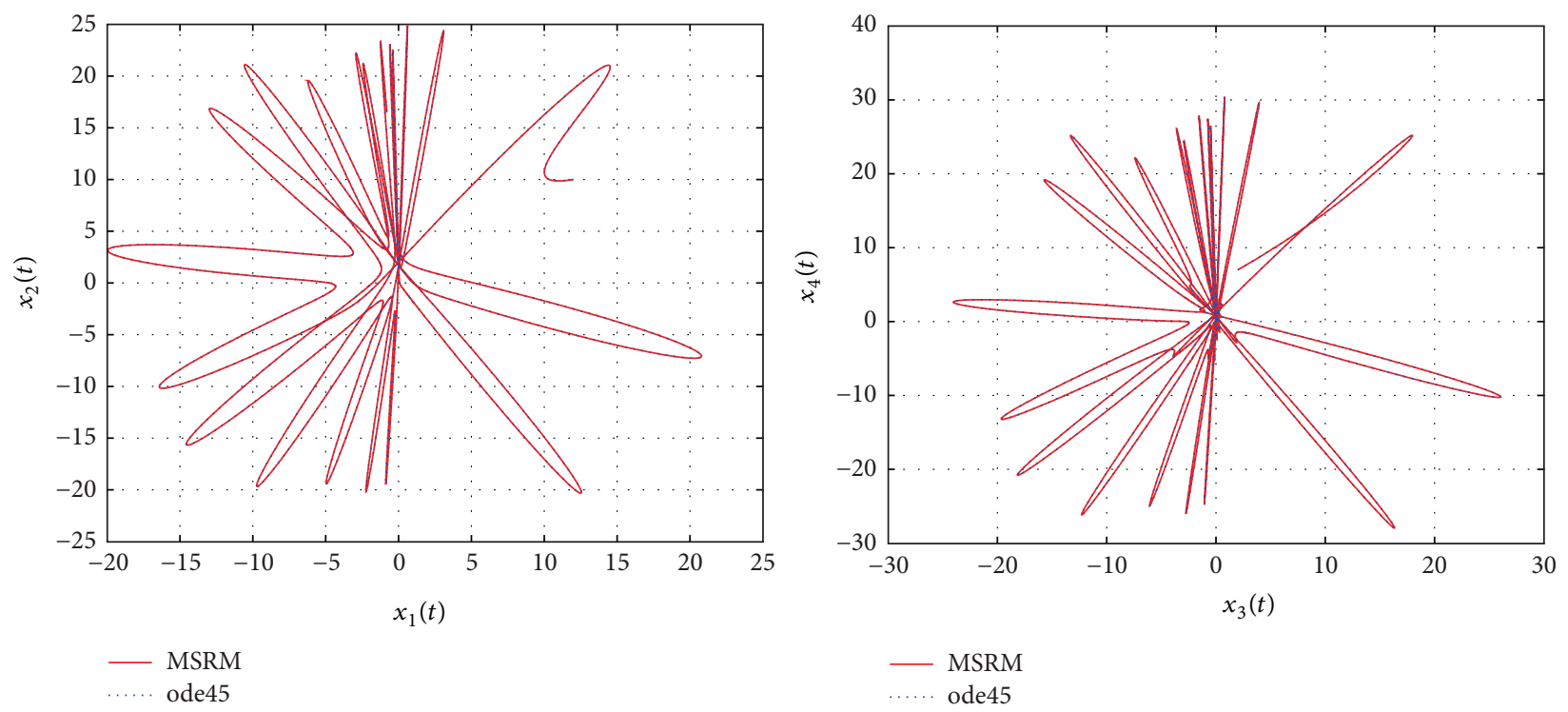

FIGURE 4: Phase portraits of the hyperchaotic complex Lorenz system.
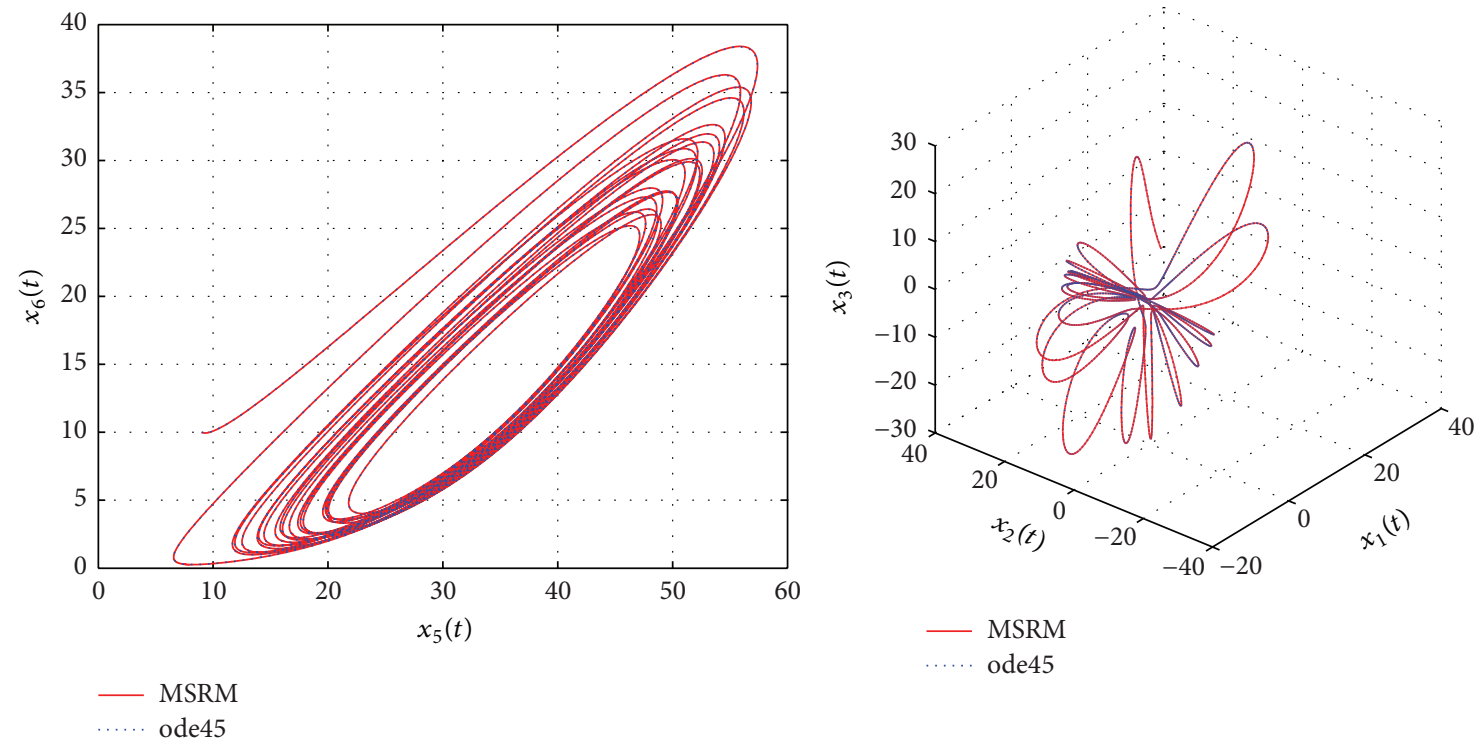

FIGURE 5: Phase portraits of the hyperchaotic complex Lorenz system.

Lyapunov exponent, two Lyapunov exponents of zero, and two negative Lyapunov exponents for system (22), which means system (22) is chaotic [24]. The values of parameters and initial values are $a=11, b=20$, and $x_{1}(0)=1, x_{2}(0)=2$, $x_{3}(0)=3, x_{4}(0)=4, x_{5}(0)=5$.

For (21), the parameters $\alpha_{r, k}$ and $f_{r}$ are defined as

$$
\begin{aligned}
\alpha_{1,1}=-a, & \alpha_{1,3}=a, & \alpha_{2,2}=-a, \\
\alpha_{2,4}=a, & \alpha_{3,1}=b, & \alpha_{3,3}=-1,
\end{aligned}
$$

$$
\begin{gathered}
\alpha_{4,2}=b, \quad \alpha_{4,4}=-1, \quad \alpha_{5,5}=-1, \\
f_{3}=-x_{1} x_{5}, \quad f_{4}=-x_{2} x_{5}, \quad f_{5}=x_{1} x_{3}+x_{2} x_{4},
\end{gathered}
$$

with all other $\alpha_{r, k}$ and $f_{r}=0$ for $r, k=1,2, \ldots, 5$.

The results obtained were compared to those from the MATLAB inbuilt solver, ode45. The ode45 solver integrates a system of ordinary differential equations using explicit 4th and 5th Runge-Kutta formula. Tables 3 and 4 show 
TABLE 3: Numerical comparison between MSRM and ode45 for the complex permanent magnet synchronous motor.

\begin{tabular}{|c|c|c|c|c|c|c|}
\hline \multirow{2}{*}{$t$} & \multicolumn{2}{|c|}{$x_{1}(t)$} & \multicolumn{2}{|c|}{$x_{2}(t)$} & \multicolumn{2}{|c|}{$x_{3}(t)$} \\
\hline & MSRM & ode 45 & MSRM & ode 45 & MSRM & ode45 \\
\hline 3 & -3.85711 & -3.85711 & -5.66683 & -5.66683 & -5.20445 & -5.20445 \\
\hline 10 & -0.33729 & -0.33729 & -0.49554 & -0.49554 & -0.49104 & -0.49104 \\
\hline 17 & 0.12630 & 0.12631 & 0.18555 & 0.18557 & 0.15550 & 0.15551 \\
\hline 24 & 0.05091 & 0.05105 & 0.07480 & 0.07501 & 0.19500 & 0.19518 \\
\hline 31 & -2.55034 & -2.54878 & -3.74694 & -3.74465 & -0.79819 & -0.79326 \\
\hline 38 & -3.93154 & -3.73551 & -5.77619 & -5.48818 & -5.33693 & -5.20595 \\
\hline
\end{tabular}

TABLE 4: Numerical comparison between MSRM and ode45 for the complex permanent magnet synchronous motor.

\begin{tabular}{lcccc}
\hline$t$ & & & & $x_{5}(t)$ \\
MSRM & $x_{4}(t)$ & ode45 & MSRM & 15.05932 \\
ode45 \\
\hline 3 & -7.64635 & -7.64635 & 10.73663 & 15.05932 \\
10 & -0.72144 & -0.72143 & 14.25582 & 14.25583 \\
17 & 0.22846 & 0.22848 & 19.33844 & 19.33921 \\
24 & 0.28649 & 0.28675 & 25.34856 & 25.35739 \\
31 & -1.17270 & -1.16545 & 14.98250 & 14.03140 \\
38 & -7.84098 & -7.64855 & \\
\hline
\end{tabular}
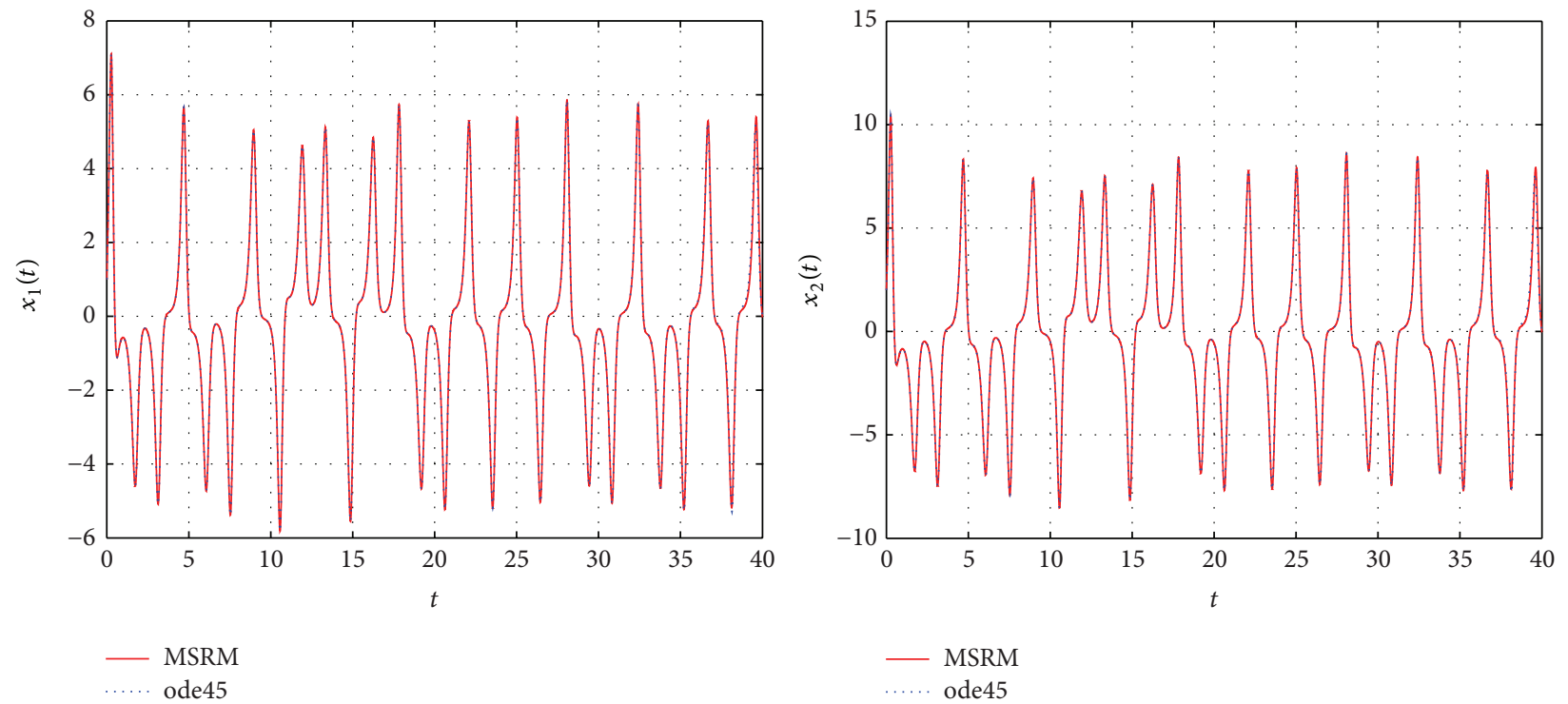

FIGURE 6: Comparison between the MSRM and ode45 results for the complex permanent magnet synchronous motor.

a comparison of the solutions of the complex permanent magnet synchronous motor computed by the MSRM and ode45. In Figures 6, 7, and 8, the MSRM graphical results are also compared with ode 45 and good agreement is observed. The MRSM phase portraits in Figures 9 and 10 were also found to be exactly the same as those computed using ode45. This shows that the proposed MSRM is a valid tool for solving the complex permanent magnet synchronous motor.

\section{Conclusion}

In this paper, we have applied a spectral method called the multistage spectral relaxation method (MSRM) for the solutions of hyperchaotic complex systems. The proposed MSRM was developed using the Gauss-Seidel idea of decoupling systems of equations and using Chebyshev pseudospectral methods to solve the resulting decoupled system on a sequence of multiple intervals. The proposed MSRM was 

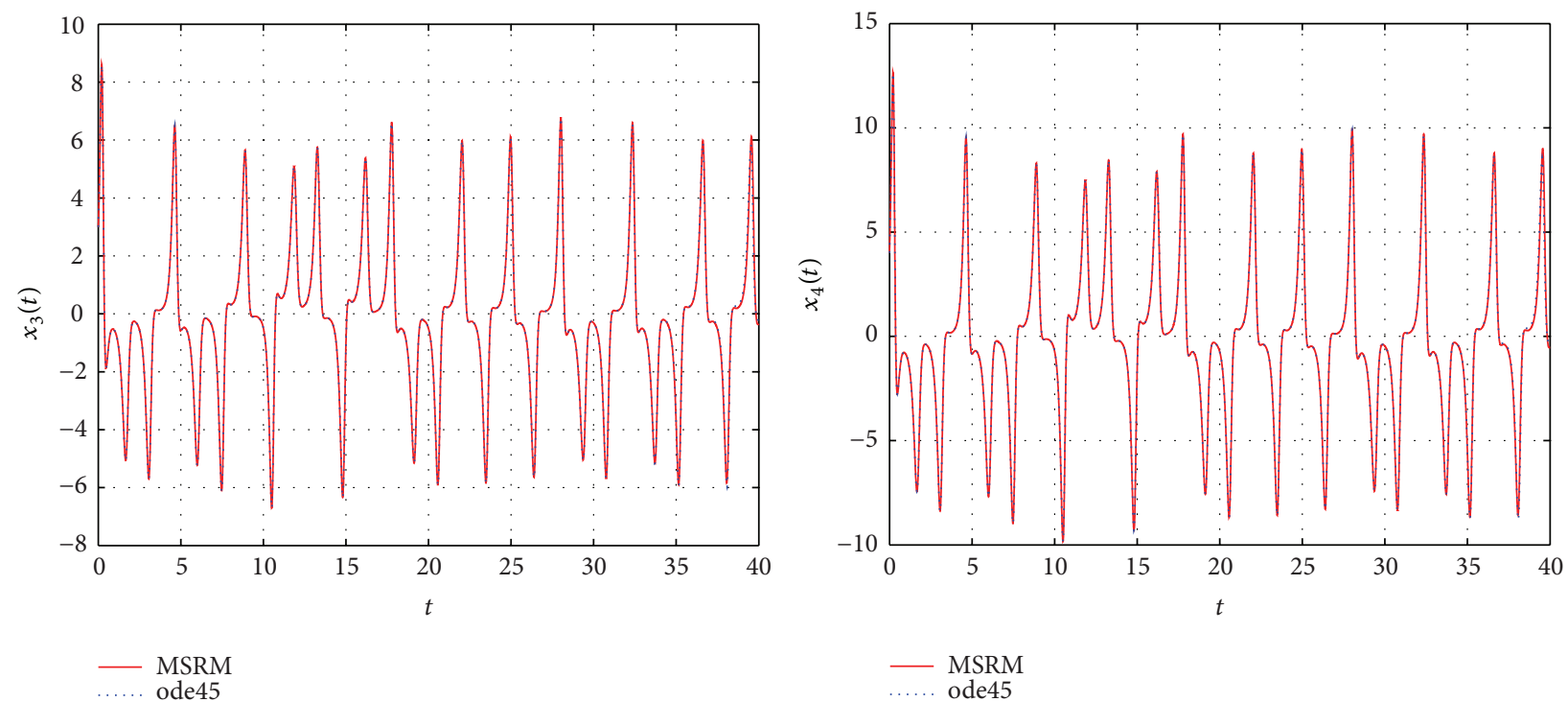

- MSRM

..... ode45

FIGURE 7: Comparison between the MSRM and ode45 results for the complex permanent magnet synchronous motor.

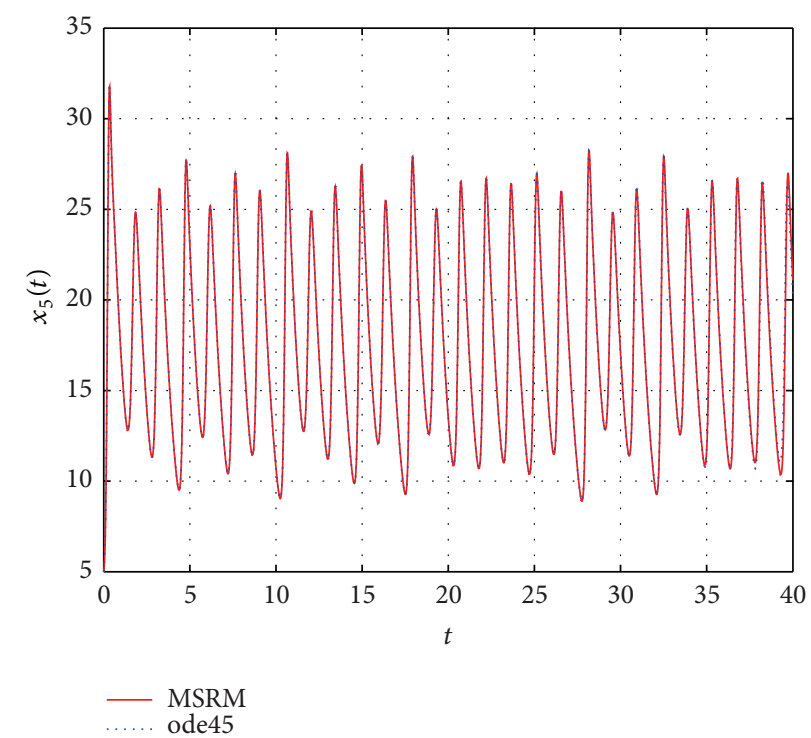

FIGURE 8: Comparison between the MSRM and ode45 results for the complex permanent magnet synchronous motor.
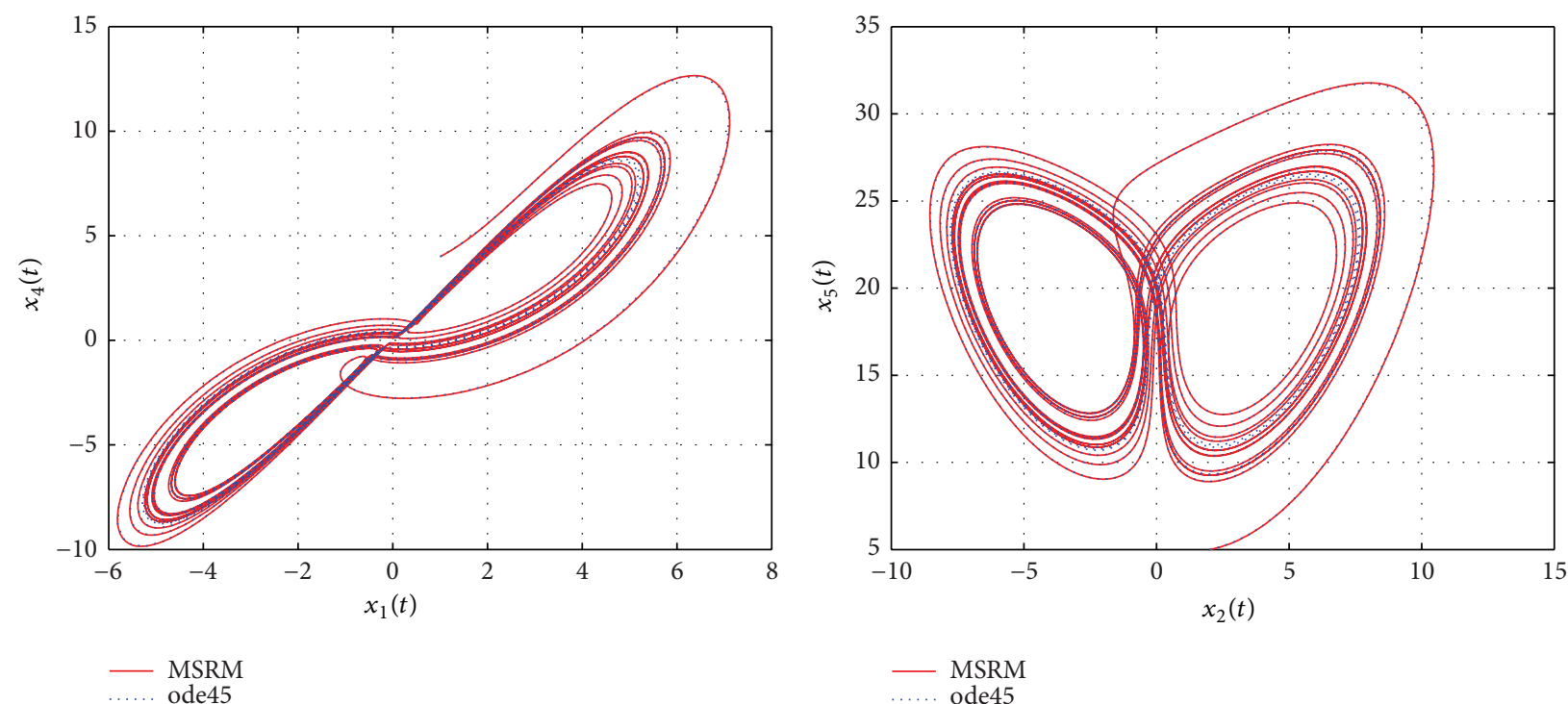

FIGURE 9: Phase portraits of the complex permanent magnet synchronous motor. 

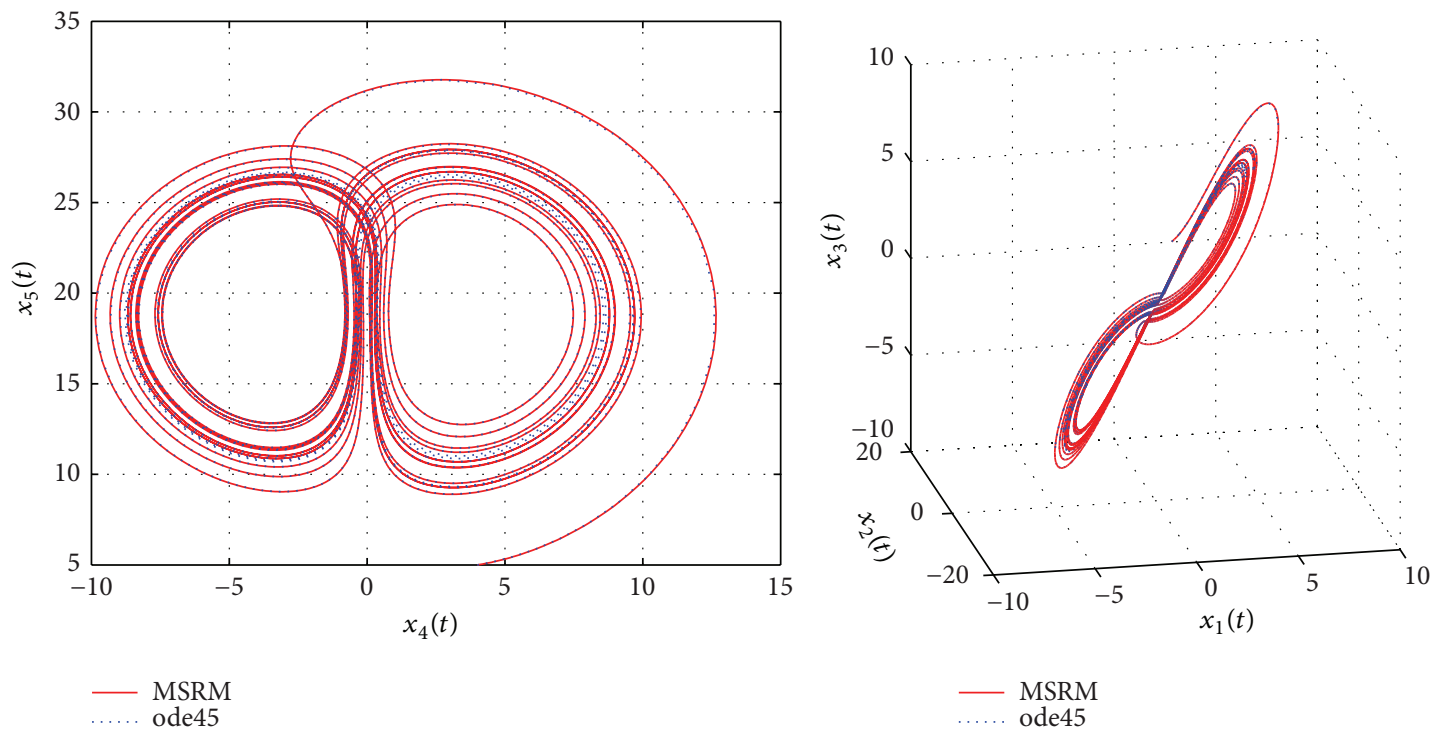

FIGURE 10: Phase portraits of the complex permanent magnet synchronous motor.

used to solve the hyperchaotic complex Lorenz system and complex permanent magnet synchronous motor. The accuracy and validity of the proposed method was tested against Matlab Runge-Kutta based inbuilt solvers and against previously published results.

\section{Conflict of Interests}

The authors declare that there is no conflict of interests regarding the publication of this paper.

\section{References}

[1] G. Chen and T. Ueta, "Yet another chaotic attractor," International Journal of Bifurcation and Chaos in Applied Sciences and Engineering, vol. 9, no. 7, pp. 1465-1466, 1999.

[2] Z. Wei, R. Wang, and A. Liu, "A new finding of the existence of hidden hyperchaotic attractors with no equilibria," Mathematics and Computers in Simulation, vol. 100, pp. 13-23, 2014.

[3] A. C. Fowler, J. D. Gibbon, and M. J. McGuinness, "The complex Lorenz equations," Physica D: Nonlinear Phenomena, vol. 4, no. 2, pp. 139-163, 1981/82.

[4] A. G. Vladimirov, V. Y. Toronov, and V. L. Derbov, "Properties of the phase space and bifurcations in the complex Lorenz model," Technical Physics, vol. 43-48, pp. 877-884, 1998.

[5] A. Rauh, L. Hannibal, and N. B. Abraham, "Global stability properties of the complex Lorenz model," Physica D: Nonlinear Phenomena, vol. 99, no. 1, pp. 45-58, 1996.

[6] R. L. Lang, "A stochastic complex model with random imaginary noise," Nonlinear Dynamics, vol. 62, no. 3, pp. 561-565, 2010.

[7] G. M. Mahmoud, M. E. Ahmed, and E. E. Mahmoud, "Analysis of hyperchaotic complex Lorenz systems," International Journal of Modern Physics C, vol. 19, no. 10, pp. 1477-1494, 2008.

[8] G. M. Mahmoud, E. E. Mahmoud, and M. E. Ahmed, "On the hyperchaotic complex Lü system,” Nonlinear Dynamics, vol. 58, no. 4, pp. 725-738, 2009.
[9] G. M. Mahmoud, M. A. Al-Kashif, and S. A. Aly, "Basic properties and chaotic synchronization of complex Lorenz system," International Journal of Modern Physics C, vol. 18, no. 2, pp. 253-265, 2007.

[10] A. K. Alomari, M. S. Noorani, and R. Nazar, "Explicit series solutions of some linear and nonlinear Schrodinger equations via the homotopy analysis method," Communications in Nonlinear Science and Numerical Simulation, vol. 14, no. 4, pp. 11961207, 2009.

[11] S. Effati, H. Saberi Nik, and M. Shirazian, "An improvement to the homotopy perturbation method for solving the HamiltonJacobi-Bellman equation," IMA Journal of Mathematical Control and Information, vol. 30, no. 4, pp. 487-506, 2013.

[12] M. S. Chowdhury and I. Hashim, "Application of multistage homotopy-perturbation method for the solutions of the Chen system," Nonlinear Analysis: Real World Applications, vol. 10, no. 1, pp. 381-391, 2009.

[13] S. M. Goh, M. S. Noorani, and I. Hashim, "On solving the chaotic Chen system: a new time marching design for the variational iteration method using Adomian's polynomial," Numerical Algorithms, vol. 54, no. 2, pp. 245-260, 2010.

[14] A. Gökdoğan, M. Merdan, and A. Yildirim, "The modified algorithm for the differential transform method to solution of Genesio systems," Communications in Nonlinear Science and Numerical Simulation, vol. 17, no. 1, pp. 45-51, 2012.

[15] H. Saberi Nik, S. Effati, S. S. Motsa, and M. Shirazian, "Spectral homotopy analysis method and its convergence for solving a class of nonlinear optimal control problems," Numerical Algorithms, vol. 65, no. 1, pp. 171-194, 2014.

[16] H. Saberi Nik, S. Effati, S. S. Motsa, and S. Shateyi, "A new piecewise-spectral homotopy analysis method for solving chaotic systems of initial value problems," Mathematical Problems in Engineering, vol. 2013, Article ID 583193, 13 pages, 2013.

[17] S. Effati, H. Saberi Nik, and A. Jajarmi, "Hyperchaos control of the hyperchaotic Chen system by optimal control design," Nonlinear Dynamics, vol. 73, no. 1-2, pp. 499-508, 2013.

[18] S. S. Motsa, P. G. Dlamini, and M. Khumalo, "Solving hyperchaotic systems using the spectral relaxation method," Abstract and Applied Analysis, vol. 2012, Article ID 203461, 18 pages, 2012. 
[19] S. S. Motsa, P. Dlamini, and M. Khumalo, "A new multistage spectral relaxation method for solving chaotic initial value systems," Nonlinear Dynamics, vol. 72, no. 1-2, pp. 265-283, 2013.

[20] L. N. Trefethen, Spectral Methods in MATLAB, SIAM, 2000.

[21] C. Luo and X. Wang, "Hybrid modified function projective synchronization of two different dimensional complex nonlinear systems with parameters identification," Journal of the Franklin Institute, vol. 350, no. 9, pp. 2646-2663, 2013.

[22] J. Yu, B. Chen, H. Yu, and J. Gao, "Adaptive fuzzy tracking control for the chaotic permanent magnet synchronous motor drive system via backstepping," Nonlinear Analysis: Real World Applications, vol. 12, no. 1, pp. 671-681, 2011.

[23] F. Zhang, C. Mu, X. Wang, I. Ahmed, and Y. Shu, "Solution bounds of a new complex PMSM system," Nonlinear Dynamics, vol. 74, no. 4, pp. 1041-1051, 2013.

[24] X. Wang and H. Zhang, "Backstepping-based lag synchronization of a complex permanent magnet synchronous motor system," Chinese Physics B, vol. 22, no. 4, Article ID 048902, 2013. 


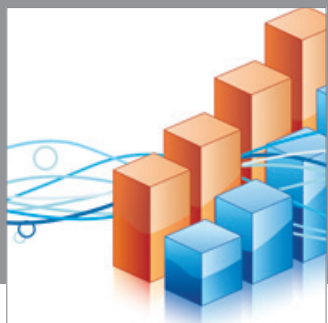

Advances in

Operations Research

mansans

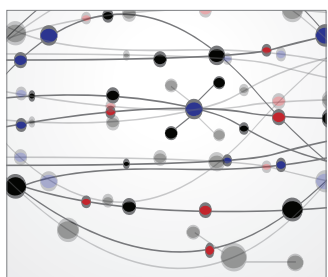

The Scientific World Journal
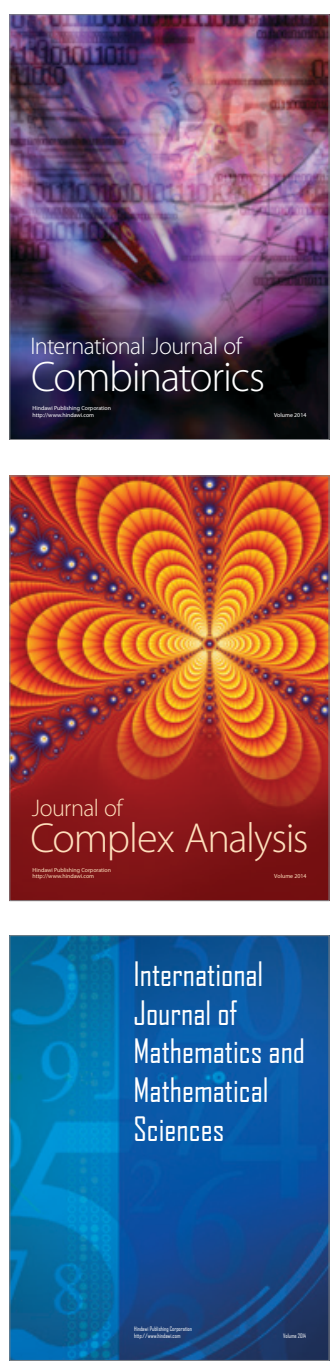
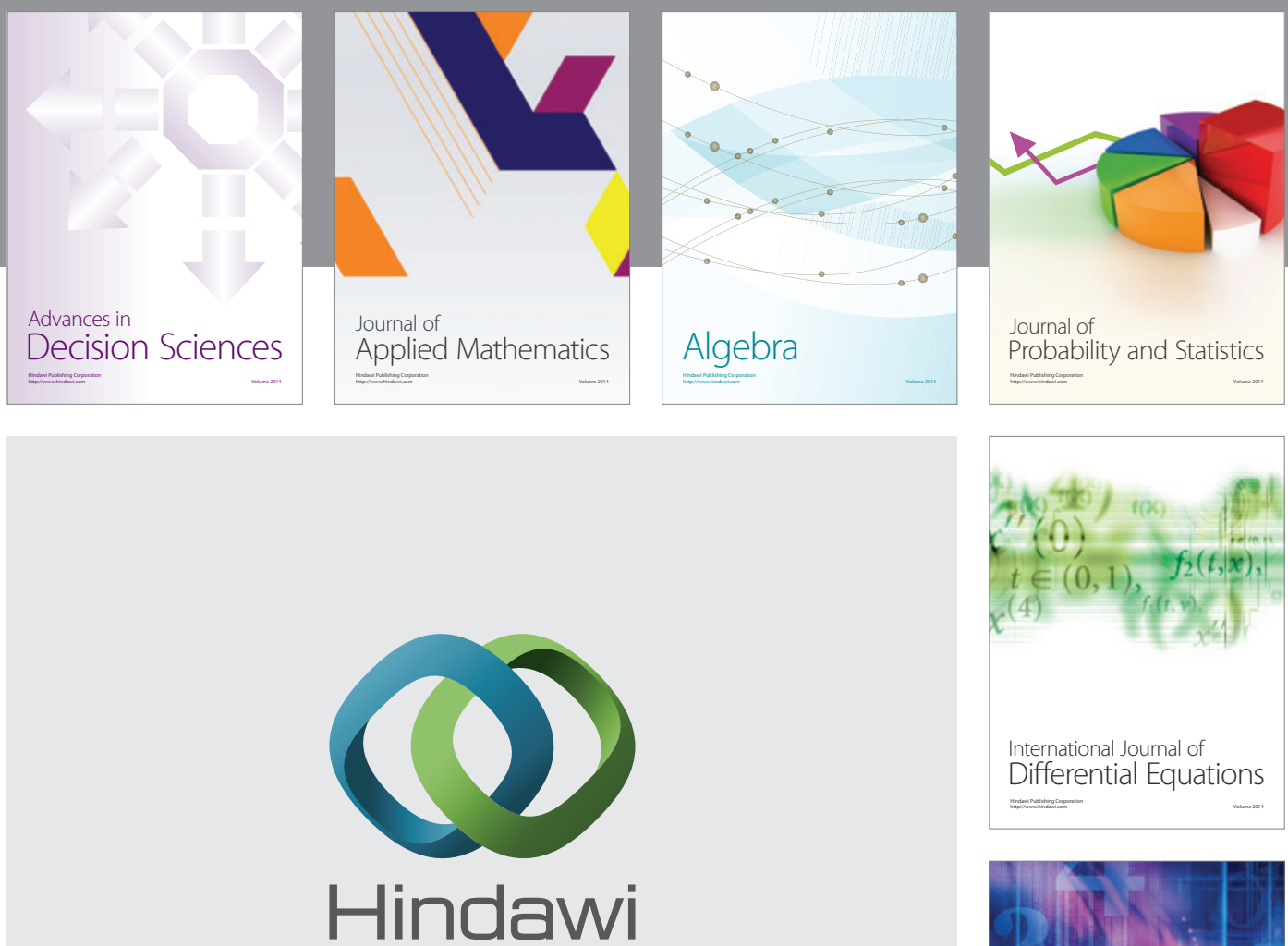

Submit your manuscripts at http://www.hindawi.com
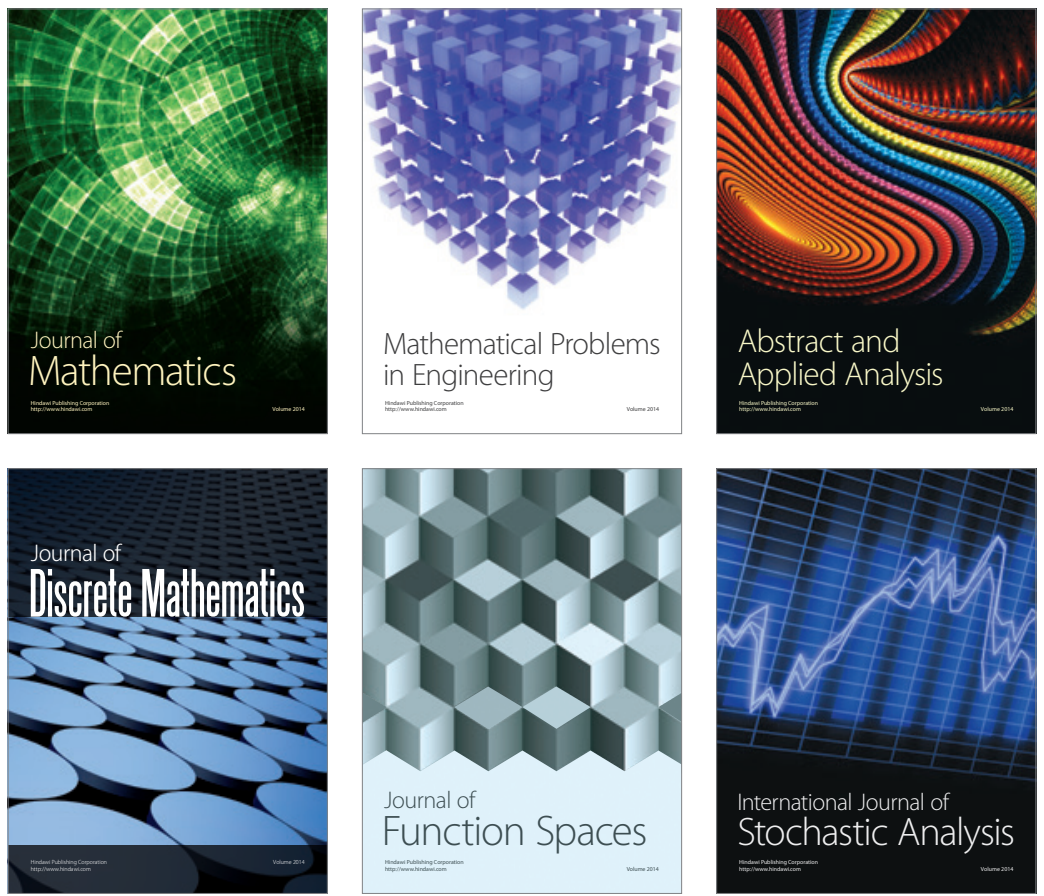

Journal of

Function Spaces

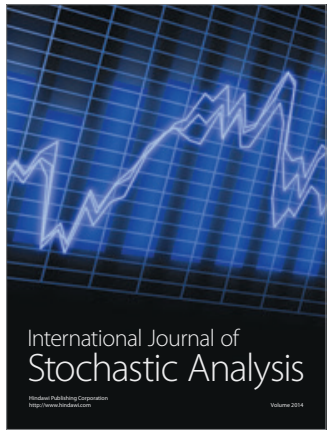

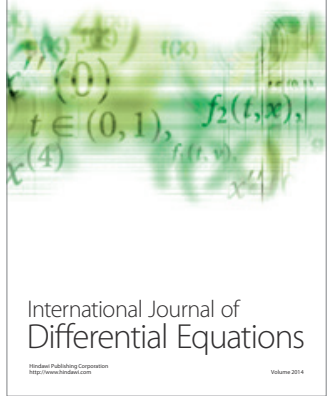
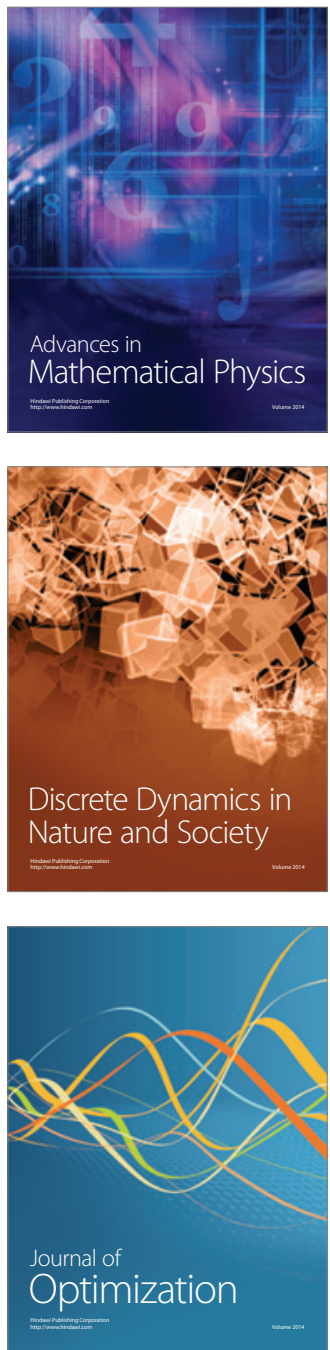